\title{
Psicologia: entre a Atuação e a Formação para o Sistema Único de Saúde
}

\author{
Raquel Antunes de Mello ${ }^{1}$ \\ ${ }^{1}$ Universidade Comunitária da Região de Chapecó, \\ SC, Brasil.
}

\author{
Carla Rosane Paz Arruda Teo ${ }^{2}$ \\ ${ }^{2}$ Universidade Comunitária da Região de Chapecó, \\ SC, Brasil.
}

Resumo: Com o desenvolvimento e implantação de diversas políticas públicas, a Psicologia vem se reconfigurando e adentrando nos espaços da saúde pública. Este artigo objetiva analisar como têm sido referidas na literatura científica as temáticas da formação e da atuação profissional do psicólogo no Sistema Único de Saúde, no nível da Atenção Básica. Para tanto, realizou-se uma pesquisa bibliográfica, descritiva, de abordagem qualitativa, fundamentada nos critérios da revisão integrativa de literatura, considerando a importância de delinear o estado da arte referente à temática estudada. A pesquisa ocorreu a partir de busca na Biblioteca Virtual em Saúde, entre dezembro de 2016 e janeiro de 2017. Foram, inicialmente, localizados 553 artigos. A partir dos critérios de inclusão e exclusão, resultou um conjunto de 22 artigos que constituiu o corpus da pesquisa. A análise dos artigos selecionados evidenciou que a prioridade dos estudos está na atuação e não na formação profissional, embora saliente-se que ambas estão intrinsecamente vinculadas. Cabe destacar que, segundo os estudos analisados, os profissionais replicam, em sua atuação, os modelos de clínica tradicional, hegemônica, apreendidos no seu processo de formação. Compreende-se como alternativas para a transformação desse cenário, mudanças na formação que abranjam desde os componentes curriculares dedicados à saúde pública e à Atenção Básica até a criação de espaços de reflexão e práticas sob o prisma da análise institucional, Psicologia social e comunitária, buscando-se a ampliar o olhar sobre o sujeito e sua subjetividade.

Palavras-chave: Atuação do psicólogo, Formação do Psicólogo, Sistema Único de Saúde.

\section{Psychology: between Practice and Training for the Unified Health System}

\begin{abstract}
With the development and implementation of several public policies, psychology has been reconfiguring itself and entering public health spaces. This article aims to analyze how training and professional performance of the psychologist in the Unified Health System, at the level of Primary Care, have been mentioned in the scientific literature. For that, a descriptive bibliographical research was carried out with a qualitative approach, based on the criteria of the integrative literature review, considering the importance of delineating the state of the art referring to the subject studied. The research occurred from a search in the Virtual Health Library between December 2016 and January 2017. Initially, 553 articles were identified. From the inclusion and exclusion criteria, a set of 22 articles resulted in the research corpus. The analysis of the selected articles showed that the priority of the studies is set in performance and not in training, although it is emphasized that both are intrinsically linked. It should be noted that, according to the studies analyzed, professionals replicate, in their work, the traditional hegemonic clinic models seized in their training process. Alternatives for the transformation of this scenario are changes in training that range from the curricular components dedicated to public health and Primary Care to the creation of spaces for reflection, and practices under the prism of institutional analysis, social psychology and community, that seek to broaden the view on the subject and his subjectivity.
\end{abstract}

Keywords: Psychologist performance, Psychologist Education, Single Health System. 


\title{
Psicología: entre la Práctica y la Capacitación para el Sistema Único de Salud
}

\begin{abstract}
Resumen: Con el desarrollo e implantación de diversas políticas públicas, la psicología se viene reconfigurando y adentrando en los espacios de la salud pública. Este artículo objetivo analizar cómo se han mencionado en la literatura científica las temáticas de la formación y actuación profesional del psicólogo en el Sistema Único de Salud, en la Atención Primaria. Se realizó una investigación bibliográfica, descriptiva, de abordaje cualitativo, fundamentada en los criterios de la revisión integrativa de literatura, considerando la importancia de delinear el estado del arte referente a la temática estudiada. La investigación ocurrió a partir de la búsqueda en la Biblioteca Virtual en Salud, entre diciembre de 2016 y enero de 2017. Fueron localizados 553 artículos. A partir de los criterios de inclusión y exclusión, resultó un conjunto de 22 artículos que constituyó el corpus de la investigación. El análisis de los artículos seleccionados evidenció que la prioridad está en la actuación y no en la formación, aunque se destaca que ambas están intrínsecamente vinculadas. Cabe destacar que, según los estudios, los profesionales replican, en su actuación, los modelos de clínica tradicional, hegemónica, incautados en su proceso de formación. Se entiende como alternativas para la transformación de ese escenario, cambios en la formación que abarcan desde los componentes curriculares dedicados a la salud pública y a la Atención Primaria hasta la creación de espacios de reflexión y prácticas bajo el prisma del análisis institucional, psicología social y social de la comunidad, buscando a ampliar la mirada sobre el sujeto y su subjetividad.
\end{abstract}

Palabras clave: Actuación del psicólogo, Formación del Psicólogo, Sistema Único de Salud.

\section{Introdução}

Refletir sobre a atuação do psicólogo no campo da saúde pública remete, incondicionalmente, à discussão acerca da formação em Psicologia e do quanto ela replica um modelo biomédico hegemônico. Nessa perspectiva, surge uma lacuna, no processo de formação, por falta de problematização, participação social crítica e atuação interdisciplinar, aspectos que constituem a subjetividade do profissional psicólogo.

A Psicologia foi regulamentada como profissão a partir de 1962, consolidando-se e sendo reconhecida, inicialmente, pela atuação em três áreas principais: clínica, organizacional e educacional (Azevedo, \& Kind, 2013). Os psicólogos, até a década de 1970, atuavam nos hospitais, em saúde mental. Em meio às reformas psiquiátrica e sanitária, esse modelo hospitalocêntrico foi questionado, dando abertura ao surgimento da clínica ampliada. Nos anos 1980, um contexto marcado pela crise econômica afetou os consultórios particulares de Psicologia, os quais atendiam exclusivamente à classe elitizada. Nesse período, começou a ser questionada a atuação da Psicologia na sociedade e, a partir disso, os psicólogos passaram a buscar novos campos de atuação (Ferreira, 2011; Souza, \& Santos, 2012).
Nesse cenário, em que vinham ocorrendo, concomitantemente, o movimento da Reforma Sanitária, a promulgação da Constituição Federal de 1988 e o estabelecimento do Sistema Único de Saúde (SUS), a saúde pública emergiu como um novo e profícuo campo de atuação para os psicólogos (Souza, \& Santos, 2012). Assim, a partir da década de 1990, os psicólogos passaram a se inserir na área da saúde pública, mas o fizeram replicando o modelo clínico tradicional, que reforça a dicotomia indivíduo-sociedade e se fundamenta em uma lógica de neutralidade da ciência. Segundo esse modelo, o sujeito e os fenômenos não são compreendidos em sua dimensão singular, social, cultural e econômica, o que se coloca em contradição aos princípios do SUS (Huning, Silva, Silva, \& Lessa, 2013).

A esse propósito, cabe destacar que o SUS instituiu o atendimento à população por meio da Atenção Primária à Saúde (APS), que representa o primeiro contato do usuário com a rede assistencial do sistema de saúde. No Brasil, adota-se a designação Atenção Básica à Saúde (ABS), a fim de enfatizar a reorientação do modelo assistencial e a contraposição à proposta político-ideológica da atenção primária seletiva. Dessa forma, a ABS reforça o princípio da universa- 
lidade da atenção e assume a Saúde da Família como estratégia principal de atuação. A ABS é, em essência, um complexo conjunto de conhecimentos e ações, que demanda uma intervenção ampla - da promoção à recuperação em saúde - e articulada aos demais níveis do sistema, priorizando a integralidade da atenção. Assim, a ABS está fundamentada em alguns elementos, como territorialização, indissociabilidade de ações preventivas e curativas, atenção centrada na família, contemplando cuidado em nível individual e coletivo, além de orientação democrática, participação comunitária e trabalho em equipe (Ministério da Saúde, 2009).

Nesse sentido, a Estratégia Saúde da Família (ESF) - que se constituiu a partir da expansão e consolidação do Programa Saúde da Família - se caracteriza como uma inovação, incorporando mudanças na concepção de saúde, no processo de trabalho e no entendimento sobre o objeto e o produto do trabalho em saúde. A ESF é o principal dispositivo de organização da ABS, no Brasil, devido aos princípios teóricos e políticos em que se ancora, representando uma via importante para a superação dos modos tradicionais de pensar e produzir saúde (Soratto, Pires, Dornelles, \& Lorenzetti, 2015). No campo da saúde pública, o Núcleo Ampliado de Saúde da Família (NASF) é uma estratégia que complementa as atividades da ABS por meio, especialmente, do apoio matricial. Composto por uma equipe multiprofissional, o NASF proporciona suporte às equipes de Saúde da Família, de forma a contribuir para sua capacidade de resolução, atuando de forma integrada, compartilhando conhecimento e experiências na solução de problemas e ampliando as ações para além das Unidades Básicas de Saúde (UBS) (Perrella, 2015; Portaria MS n- 2.436, 2017). Por sua natureza inovadora, integrada e coletiva, o modelo de trabalho do NASF é desafiador para os profissionais da saúde em geral, pois contrapõe o modelo biomédico dominante em que, historicamente, se fundamentaram não só a atenção à saúde da população como, também, os processos de formação profissional, que não preparam para o compartilhamento de saberes e o trabalho interdisciplinar (Leite, Nascimento, \& Oliveira, 2014; Perrella, 2015).

No que tange à inserção da Psicologia nesse contexto, um marco importante na dimensão da formação foi a articulação entre os Ministérios da Saúde e da Educação, que resultou, em 2004, na implantação das Diretrizes Curriculares Nacionais (DCN) e no reconhecimento da Psicologia como um curso da área da saúde, ou seja, como parte de um contexto em que se tinha o objetivo de aproximar os cursos de formação das demandas do serviço. Nesse momento, as mudanças curriculares incluíram disciplinas de saúde pública na graduação e fundamentaram-se nos princípios do SUS, no que diz respeito às competências e habilidades previstas para a formação de profissionais de saúde, contemplando dispositivos de ensino-aprendizagem interdisciplinares (Pró-Saúde, Pet-Saúde, Ver-SUS, residências multiprofissionais), em uma dinâmica de integração ensino-serviço (Ministério da Saúde, 2009).

Contudo, espaços da saúde pública são, ainda, ocupados de forma tímida pela Psicologia como cenários de aprendizagem nos processos de formação, com reflexos na atuação dos egressos. Tem sido proposto que o perfil generalista estabelecido pelas DCN, ao abranger diversas áreas - como educação, saúde, assistência, trabalho, avaliação e clínica -, repercute em pulverização dos cenários educativos e em prejuízo do adensamento da formação em saúde pública (Batista, Carmona, \& Fonseca, 2014). Para Carvalho e Ceccim (2009), um processo de formação adequado para o campo da saúde pública deve contemplar o aumento dos espaços de aprendizagem, com vistas ao desenvolvimento, pelos acadêmicos, de habilidades para gestão, atenção à saúde e controle social, utilizando metodologias ativas e incentivando a atuação multiprofissional. Manoel, Combinato, Gomes e Silva (2014) reforçam essas ponderações, afirmando que a formação dos profissionais da área da saúde precisa ir além do conhecimento técnico de caráter biomédico, de forma que seja promovido o desenvolvimento de habilidades para o trabalho multiprofissional e interdisciplinar (Manoel et al., 2014).

Ainda considerando a inserção do psicólogo no campo da saúde pública, pondera-se que o SUS se destaca entre os maiores contratadores do profissional no País, fator que pode contribuir para reconfigurar as diretrizes da formação nesta área e, por consequência, a atuação da Psicologia (Ferreira, 2011). Nesse sentido, tanto a formação quanto a atuação do psicólogo no SUS, especialmente no nível da Atenção Básica, são campos profícuos para pesquisas que possam resultar em fortalecimento da integração ensino-serviço-comunidade e, consequentemente, em novas práticas de cuidado em saúde, que estejam efetivamente a serviço da vida (Perrella, 2015). 
Diante dessas questões, o presente estudo teve por objetivo analisar como têm sido referidas na literatura científica as temáticas da formação e da atuação profissional do psicólogo no SUS, no nível da Atenção Básica.

\section{Método}

Trata-se de um estudo bibliográfico, descritivo, fundamentado nos critérios da revisão integrativa de literatura. Para realizar a pesquisa, consideramos a importância de delinear o estado da arte sobre a formação e a atuação profissional do psicólogo no SUS, no nível da Atenção Básica.
A pesquisa na literatura científica ocorreu a partir de busca no Portal Regional da Biblioteca Virtual em Saúde (BVS), selecionando o item busca avançada e utilizando os seguintes descritores e termos: "Educação Continuada", "Psicologia", "Sistema Único de Saúde", "Atenção Primária à Saúde", "Saúde Pública”, "Educação Superior", "Educação Profissional em Saúde Pública”, "Formação Profissional”, "NASF”, "Prática Profissional", "Atuação Profissional" (Tabela 1).

A busca dos estudos aconteceu entre dezembro de 2016 e janeiro de 2017. Para a seleção, foram aplicados os seguintes critérios de inclusão: artigos originais ou relatos de experiência, publicados no período de

Tabela 1

Síntese do percurso de busca dos estudos para revisão integrativa de literatura sobre a formação e a atuação profissional do psicólogo no Sistema Único de Saúde, no nível da Atenção Básica, 2006-2016.

\begin{tabular}{|c|c|}
\hline Cruzamentos dos descritores e termos & $\begin{array}{c}\text { Total de estudos } \\
\text { localizados }\end{array}$ \\
\hline "Educação Continuada" and "Psicologia" and "Sistema Único de Saúde" & 4 \\
\hline “Educação Continuada" and "Psicologia” and "Atenção Primária à Saúde" & 71 \\
\hline “Educação Continuada” and “Psicologia” and “Saúde Pública” & 128 \\
\hline “Educação Superior” and “Psicologia” and "Sistema Único e Saúde” & 3 \\
\hline “Educação Superior" and "Psicologia” and “Atenção Primária à Saúde" & 1 \\
\hline “Educação Superior” and "Psicologia” and "Saúde Pública” & 10 \\
\hline “Educação Profissional em Saúde Pública” and "Psicologia” & 21 \\
\hline "Formação Profissional” and “Psicologia" and "Sistema Único de Saúde" & 18 \\
\hline “Formação Profissional” and “Psicologia” and “Atenção Primária à Saúde” & 24 \\
\hline “Formação Profissional” and "Psicologia” and “Saúde Pública” & 66 \\
\hline “Psicologia" and “NASF” & 23 \\
\hline "Prática Profissional” and "Psicologia" and "Sistema Único de Saúde" & 17 \\
\hline “Prática Profissional” and “Psicologia” and “Atenção Primária à Saúde” & 34 \\
\hline “Prática Profissional” and “Psicologia” and “Saúde Pública” & 68 \\
\hline “Atuação Profissional” and “Psicologia” and "Saúde Pública” & 30 \\
\hline "Atuação Profissional" and "Psicologia" and "Atenção Primária à Saúde" & 32 \\
\hline “Atuação Profissional” and "Psicologia” and “Sistema Único de Saúde” & 3 \\
\hline Total de estudos & 553 \\
\hline Total de estudos pré-selecionados após aplicação dos filtros & 181 \\
\hline Estudos não disponíveis & 38 \\
\hline Estudos duplicados & 56 \\
\hline Não tinham relação com o tema proposto (baixa aderência) & 61 \\
\hline Não era artigo original ou relato de experiência & 4 \\
\hline Total de estudos selecionados - incluídos na pesquisa & 22 \\
\hline
\end{tabular}

Fonte: Elaboração das autoras (2017). 
2006 a 2016, nos idiomas português, inglês ou espanhol, disponíveis on-line em texto completo de livre acesso. Uma primeira triagem foi feita a partir dos títulos. Na sequência, foram aplicados os seguintes critérios de exclusão: publicações duplicadas e/ou com baixa aderência ao objetivo desta revisão (identificada por leitura flutuante).

Da aplicação dos critérios de inclusão e exclusão descritos, derivou um conjunto de 22 artigos (Tabela 2) que constituiu o corpus da pesquisa.

Tabela 2

Artigos selecionados para revisão integrativa da literatura sobre a formação e a atuação profissional do psicólogo no Sistema Único de Saúde, no nível da Atenção Básica, 2006-2016.

\begin{tabular}{|c|c|c|c|c|}
\hline$E^{*}$ & Ano & Autores & Título & Periódico \\
\hline E1 & 2015 & Cela, e Oliveira & $\begin{array}{l}\text { O psicólogo no Núcleo de Apoio à Saúde da } \\
\text { Família: articulação de saberes e ações }\end{array}$ & Estudos de Psicologia \\
\hline E2 & 2015 & Perrella & $\begin{array}{l}\text { A experiência da Psicologia no NASF: capturas, } \\
\text { embates e invenções capturas }\end{array}$ & $\begin{array}{l}\text { Revista } \\
\text { Interinstitucional de } \\
\text { Psicologia }\end{array}$ \\
\hline E3 & 2015 & $\begin{array}{l}\text { Gomes, e } \\
\text { Pedrero }\end{array}$ & $\begin{array}{l}\text { Queixa escolar: encaminhamentos e atuação } \\
\text { profissional em um município do interior } \\
\text { paulista }\end{array}$ & $\begin{array}{l}\text { Psicologia: Ciência e } \\
\text { Profissão }\end{array}$ \\
\hline E4 & 2015 & $\begin{array}{l}\text { Sousa, Oliveira, e } \\
\text { Costa }\end{array}$ & $\begin{array}{l}\text { Entre o especialíssimo e o apoio: psicólogos no } \\
\text { Núcleo de Apoio à Saúde da Família }\end{array}$ & Psicologia USP \\
\hline E5 & 2014 & $\begin{array}{l}\text { Batista, Carmona, e } \\
\text { Fonseca }\end{array}$ & $\begin{array}{l}\text { Formação em saúde e os cenários de } \\
\text { aprendizagem no curso de Psicologia }\end{array}$ & Psicologia Argumento \\
\hline E6 & 2014 & $\begin{array}{l}\text { Alves, Gaião, } \\
\text { Santos, e Soares }\end{array}$ & $\begin{array}{l}\text { Proposta de Estágio Supervisionado para } \\
\text { atuação de psicólogos na Saúde Pública }\end{array}$ & $\begin{array}{l}\text { Revista da Abordagem } \\
\text { Gestáltica }\end{array}$ \\
\hline E7 & 2014 & Leite et al. & $\begin{array}{l}\text { Qualidade de vida no trabalho de profissionais } \\
\text { do NASF no município de São Paulo }\end{array}$ & $\begin{array}{l}\text { Physis Revista de } \\
\text { Saúde Coletiva }\end{array}$ \\
\hline E8 & 2013 & $\begin{array}{l}\text { Hüning, Silva, Silva, } \\
\text { e Lessa }\end{array}$ & $\begin{array}{l}\text { Formação para SUS nos cursos de Psicologia em } \\
\text { Alagoas }\end{array}$ & Psicologia em Estudo \\
\hline E9 & 2013 & Azevedo, e Kind & $\begin{array}{l}\text { Psicologia nos Núcleos de Apoio à Saúde da } \\
\text { Família em Belo Horizonte }\end{array}$ & $\begin{array}{l}\text { Psicologia: Ciência e } \\
\text { Profissão }\end{array}$ \\
\hline E10 & 2013 & $\begin{array}{l}\text { Leite, Andrade, e } \\
\text { Bosi }\end{array}$ & $\begin{array}{c}\text { A inserção da Psicologia nos Núcleos de Apoio à } \\
\text { Saúde da Família }\end{array}$ & $\begin{array}{l}\text { Physis Revista de } \\
\text { Saúde Coletiva }\end{array}$ \\
\hline E11 & 2013 & $\begin{array}{l}\text { Lancman, } \\
\text { Gonçalves, } \\
\text { Cordone, e Barros, }\end{array}$ & $\begin{array}{l}\text { Estudo do trabalho e do trabalhar no Núcleo de } \\
\text { Apoio à Saúde da Família }\end{array}$ & $\begin{array}{l}\text { Revista de Saúde } \\
\text { Pública }\end{array}$ \\
\hline E12 & 2012 & $\begin{array}{l}\text { Archanjo, e } \\
\text { Schraiber }\end{array}$ & $\begin{array}{l}\text { A atuação dos psicólogos em Unidades Básicas } \\
\text { de Saúde na cidade de São Paulo }\end{array}$ & Saúde \& Sociedade \\
\hline E13 & 2012 & $\begin{array}{l}\text { Guareschi, Machry, } \\
\text { Reis, Dhein, e } \\
\text { Bennemann }\end{array}$ & $\begin{array}{c}\text { Implicações das áreas biomédicas da formação } \\
\text { do psicólogo no SUS }\end{array}$ & Psicologia Argumento \\
\hline E14 & 2012 & Santeiro & $\begin{array}{l}\text { Processos clínicos em Núcleos de Apoio à Saúde } \\
\text { da Família/NASF: Estágio Supervisionado }\end{array}$ & $\begin{array}{l}\text { Psicologia: Ciência e } \\
\text { Profissão }\end{array}$ \\
\hline E15 & 2011 & $\begin{array}{l}\text { Guareschi, } \\
\text { Reis, Dhein, } \\
\text { Bennemann, e } \\
\text { Machry }\end{array}$ & $\begin{array}{c}\text { A avaliação psicológica, psicopatologia e as } \\
\text { psicoterapias na formação do profissional de } \\
\text { saúde para o SUS: um estudo dos currículos dos } \\
\text { cursos de Psicologia }\end{array}$ & $\begin{array}{l}\text { Revista Mal-estar e } \\
\text { Subjetividade }\end{array}$ \\
\hline E16 & 2011 & Gonze, e Silva & $\begin{array}{l}\text { A integralidade na formação dos profissionais } \\
\text { de saúde: tecendo valores }\end{array}$ & $\begin{array}{l}\text { Physis Revista de } \\
\text { Saúde Coletiva }\end{array}$ \\
\hline
\end{tabular}

Continua 
Continuação

\begin{tabular}{|c|c|c|c|c|}
\hline E17 & 2011 & Santos, e Castro & Demanda por grupos, Psicologia e controle & Psicologia e Sociedade \\
\hline E18 & 2010 & Sundfeld & $\begin{array}{l}\text { Clínica ampliada na atenção básica e processos } \\
\text { de subjetivação: relato de uma experiência }\end{array}$ & $\begin{array}{l}\text { Physis Revista de } \\
\text { Saúde Coletiva }\end{array}$ \\
\hline E19 & 2009 & $\begin{array}{l}\text { Araujo, Almeida, e } \\
\text { Guimarães }\end{array}$ & $\begin{array}{l}\text { Reflexões sobre o ensino de Psicologia fora } \\
\text { da academia a partir de um curso destinado à } \\
\text { comunidade }\end{array}$ & $\begin{array}{l}\text { Transformações em } \\
\text { Psicologia }\end{array}$ \\
\hline E20 & 2009 & $\begin{array}{l}\text { Macedo, e } \\
\text { Dimenstein }\end{array}$ & $\begin{array}{l}\text { Psicologia e a produção do cuidado no campo } \\
\text { do bem-estar social }\end{array}$ & Psicologia e Sociedade \\
\hline E21 & 2009 & $\begin{array}{l}\text { Prates, e } \\
\text { Nunes }\end{array}$ & $\begin{array}{l}\text { A (re) construção do lugar do psicólogo na } \\
\text { saúde pública: das quatro paredes do Centro de } \\
\text { Saúde para os Lares }\end{array}$ & $\begin{array}{l}\text { Pesquisas e Práticas } \\
\text { Psicossociais }\end{array}$ \\
\hline E22 & 2008 & Ferreira & $\begin{array}{c}\text { Intervenção psicossocial em saúde e formação } \\
\text { do psicólogo }\end{array}$ & Psicologia e Sociedade \\
\hline
\end{tabular}

*E: estudo

Fonte: Elaboração das autoras (2017).

Estes artigos foram lidos na íntegra de forma detalhada e aprofundada, extraindo-se deles as informações que alimentaram uma matriz analítica composta pelos seguintes itens: ano de publicação, periódico, local, autores, título, palavras-chave, categoria do artigo, tipo de estudo, abordagem de pesquisa, objetivo do estudo, estrutura metodológica, principais resultados e principais conclusões. A seguir, por meio da matriz analítica construída, foram categorizadas as informações que estavam alinhadas aos objetivos da revisão e que, portanto, se apresentaram como relevantes para o estudo, compondo-se uma síntese narrativa do conhecimento sobre o tema proposto, a qual é apresentada na próxima seção.

\section{Resultados e Discussão}

\section{Caracterização do corpus da pesquisa}

Inicialmente, destaca-se que 14 dos 22 estudos selecionados foram publicados após 2011, ano de publicação das mais recentes DCN para cursos de graduação em Psicologia. Essa concentração de estudos sobre a formação e atuação profissional do psicólogo no SUS, no nível da Atenção Básica, evidencia a ênfase crescente conferida à temática e indica sua relevância e atualidade. Propõe-se que a reformulação das DCN deu novo impulso à discussão sobre os campos de atuação do psicólogo, despertando o interesse de pesquisadores sobre as práticas exer- cidas por esses profissionais e, consequentemente, sobre sua formação para a saúde pública. Na mesma direção, cabe mencionar que os primeiros estudos selecionados nesta pesquisa datam de 2008, sendo possível supor que esse fato tenha relação com a publicação da Portaria $n^{\circ}$ 154/2008 (Portaria ${ }^{\circ}$ GM $154,2008)$, que criou os NASF.

Quanto às áreas de publicação, 16 dos estudos selecionados foram publicados em periódicos da Psicologia e seis o foram em revistas da área da saúde, incluindo aquelas com foco na saúde coletiva e outras com perspectivas interdisciplinares. Entre os estudos publicados em revistas da área da Psicologia, três foram localizados em periódicos com foco em abordagens mais especialistas, como saúde mental, fenomenologia e Gestalt. Essas observações reforçam que a Psicologia continua representando um vasto campo a ser pesquisado de forma interdisciplinar, especialmente em suas interfaces com a saúde pública e coletiva, o que pode contribuir para a construção e disseminação de conhecimento sobre a inserção do psicólogo neste espaço, assim como para aproximar estes nichos de saber.

No processo de caracterizar as produções selecionadas para a revisão, foram analisadas as palavras-chave indicadas por seus autores com vistas à indexação (Tabela 3). A esse respeito, constatou-se que o termo "atuação do psicólogo" teve maior frequência, com seis citações, do que as composições que utilizaram a palavra formação ("formação" e 'formação profissional"), com quatro indicações. 


\section{Tabela 3}

Frequência das palavras-chave citadas nos artigos selecionados para a revisão integrativa da literatura sobre a formação e a atuação profissional do psicólogo no Sistema Único de Saúde, no nível da Atenção Básica, 2006-2016.

\begin{tabular}{lc}
\hline Palavras-chave & Frequência \\
\hline Psicologia & 11 \\
Atuação do Psicólogo & 6 \\
NASF & 5 \\
Atenção Básica à Saúde, Processos de Subjetivação, Saúde da Família, Subjetividade, & 4 \\
Formação ou Formação Profissional & 3 \\
Saúde mental, Atenção Primária à Saúde, Políticas Públicas de Saúde, Profissional da & \\
Saúde, Psicologia da Saúde ou Pública, SUS & 2 \\
Análise Institucional, Currículo, Graduação em Psicologia, História da Psicologia, \\
Intervenção Psicossocial, Programa Saúde da Família \\
Atenção Primária à Saúde, Biopolítica, Capacitação Profissional, Dificuldades Escolares, \\
Dinâmica de Grupo, Educação Superior, Ergonomia, Estágio Clínico, Ética, Famílias de \\
Acamados, Foucault, Orientação Vocacional, Psicologia Social, Pesquisa Qualitativa, \\
Interdisciplinaridade, Intersetorialidade, Psicologia Aplicada, Clinica Ampliada, \\
Psicologia Clínica, Qualidade de Vida no Trabalho, Serviço de Saúde, Singularidade, \\
Sociedade, Controle, Trabalho
\end{tabular}

Fonte: Elaboração das autoras (2017).

Os objetivos das produções que foram elencadas para fazer parte do arcabouço teórico da revisão apresentaram relativa homogeneidade, corroborando o índice e a prevalência das palavras-chave em destaque. Parte dos estudos apresentaram objetivos ligados à abordagem da atuação do psicólogo no SUS, no nível da Atenção Básica, incluindo os espaços das UBS e dos NASF, interessados, basicamente, em problematizar, compreender, caracterizar e/ou analisar essa atuação. Outros estudos colocaram a centralidade em aspectos da formação do psicólogo para a área da saúde, com objetivos dedicados a caracterizar, analisar, discutir, compreender e/ou intervir.

A análise das palavras-chave e dos objetivos dos estudos selecionados evidenciou a ênfase colocada na perspectiva da atuação do psicólogo na Atenção Básica. Pondera-se que essas pesquisas não priorizaram a formação como constituição principal e pano de fundo na subjetividade do profissional, aspectos raramente mencionados.

Da caracterização dos estudos selecionados, em consonância com os objetivos desta revisão, emergiram as categorias analíticas: Atuação do psicólogo no SUS, no nível da Atenção Básica e Formação do psicólogo para a atuação no SUS, no nível da Atenção Básica, segundo as quais foi construída a síntese narrativa apresentada a seguir.

\section{Atuação do psicólogo no SUS, no nível da Atenção Básica}

No campo das políticas públicas, o SUS foi pioneiro como espaço expressivo de inserção de psicólogos, o que se mantém até a atualidade (Oliveira et al., 2017). Em se tratando da ABS, em 1992, por meio da Portaria $\mathrm{n}^{\circ}$ 224, do Ministério da Saúde (1992), já ficava estabelecido um modelo de serviço de saúde mental inovador, em bases locais e comunitárias, prevendo atendimento individual e coletivo, visitas domiciliares e atividades com a comunidade (Portaria SAS/MS n ${ }^{\circ} 224,1992$; Costa, Siqueira, Uhr, Silva, \& Molinaro, 2011; Jimenez, 2011). Contudo, diante de uma inserção profissional traduzida, predominantemente, como práticas psicológicas afiliadas à clínica tradicional, inúmeras foram as críticas no sentido de que a atuação do psicólogo não contemplava os princípios do SUS (Jimenez, 2011).

Nesse contexto, tanto a criação do Programa Saúde da Família (em 1994) quanto sua consolidação, como ESF (em 2006), não incluíram o psicólogo como parte da equipe mínima na $\mathrm{ABS}$, privilegiando, contraditoriamente, uma configuração de equipe que, de certa forma, denuncia uma concepção de saúde centrada na dimensão biológica do cuidado (Scarcelli, \& Junqueira, 2011). Apenas com a criação dos NASF, em 2008, a Psicologia volta ao marco legal da ABS, 
na área estratégica da saúde mental (Jimenez, 2011; Oliveira et al., 2017).

Considerando o percurso da Psicologia na ABS - aqui, brevemente abordado -, importa conhecer o que aponta a literatura sobre o tema de sua atuação nesse cenário. Para tanto, os artigos selecionados e que fundamentam essa categoria temática são: E1, E2, E3, E4, E7, E9, E10, E11, E12, E17 E18, E19, E20, E22.

Em estudo relativamente recente (E1), Cela e Oliveira (2015) realizaram um resgate histórico sobre a redemocratização da Reforma Sanitária, em 1980, que fomentou a proposta da saúde coletiva, com vistas à transformação no contexto social e à autonomia dos sujeitos. O objetivo do estudo foi problematizar a prática dos profissionais de Psicologia nos NASF, no estado do Rio Grande do Norte, com foco na articulação de saberes na equipe multiprofissional e nas ações desenvolvidas com outras equipes e instituições da rede de atenção. As autoras ponderaram que a prática da Psicologia no NASF exige que o profissional atue de forma interdisciplinar, atendendo aos usuários na família e na comunidade, ultrapassando os limites de seu campo de saber específico. No entanto, fizeram a ressalva de que a formação em Psicologia não desenvolve essas habilidades e não instiga ao compromisso social. Ademais, os resultados do estudo evidenciaram fragilidades na articulação interdisciplinar da equipe do NASF, débil integração desta equipe com a rede e pouca atuação em apoio matricial às equipes de Saúde da Família. As autoras concluíram que a Psicologia, predominantemente, vem se materializando, no NASF, ainda de forma tradicional, revelando dificuldade de transformação de suas práticas. Porém, destacaram que, na aproximação dos profissionais com a realidade social, alguns avanços têm sido conquistados e precisam ser fortalecidos, dada sua possibilidade de promover rupturas importantes e contribuir para o campo da saúde pública (Cela, \& Oliveira, 2015).

Já o estudo (E2) realizado por Perrella (2015) teve como proposta apresentar a experiência vivida pela autora, psicóloga que atua no NASF, em um município de pequeno porte no interior da Bahia, problematizando a intervenção do profissional, a partir das diretrizes e propostas de trabalho: apoio matricial, clínica ampliada e projeto terapêutico singular. Neste estudo, foi concluído que as equipes refletiram sobre as interferências da atuação do psicólogo com os usuários, tendo havido mudanças nos modos de vida, percebendo-se a singularidade, a subjetividade e desenvolvendo-se a autonomia dos sujeitos. Porém, a autora ponderou que as equipes do NASF ainda têm dificuldades de ampliar a dimensão de seus saberes específicos, compartilhando conhecimentos e realizando planejamento em uma perspectiva integrada à equipe multiprofissional e, sobretudo, aos usuários (Perrella, 2015).

No estudo (E3) de Gomes e Pedrero (2015), o objetivo foi caracterizar o perfil da demanda por queixa escolar encaminhada às UBS de um município do interior de São Paulo, a fim de identificar e analisar o tipo de atendimento realizado pelos profissionais de Psicologia. Foi constatado que a maioria dos encaminhamentos se referia a dificuldades de aprendizagem e problemas de comportamento. As autoras concluíram que os psicólogos reproduziram, nesse contexto, o modelo clínico clássico, com atendimento individualizado e focado na problemática, atribuindo essa prática tradicional a processos de formação conservadores, fundamentados na psicanálise (Gomes, \& Pedrero, 2015).

Leite et al. (2014) desenvolveram uma pesquisa (E7) com o objetivo de identificar as percepções acerca da qualidade de vida no trabalho dos profissionais das equipes de NASF do município de São Paulo (SP). Participaram da pesquisa 40 profissionais, dos quais cinco eram psicólogos, integrantes de cinco NASF diferentes. As autoras concluíram que falta de espaço físico, dificuldades na relação interpessoal, sobrecarga de trabalho, autonomia e maturidade profissional eram fatores geradores de estresse e adoecimento dos profissionais do NASF (Leite et al., 2014).

O estudo E4 abordou o perfil e a atuação dos psicólogos do NASF em dois municípios do estado do Rio Grande do Norte. Os autores concluíram que a atuação dos psicólogos nos municípios avaliados permitia identificá-los como "apoiadores" ou "especialistas". A ideia do atendimento em clínica individualizada, caracterizando uma atuação em nível de especialidade, esteve presente tanto entre usuários, gestores, equipe do NASF e da ESF quanto entre os próprios psicólogos, que evidenciaram não considerar sua implicação social com as políticas públicas nesse contexto. Os autores defenderam que a grande mudança a ser empreendida, em termos da atuação do psicólogo no campo da saúde pública, é a de sair do lugar de "especialista" para ocupar o de "apoiador" (Sousa, Oliveira, \& Costa, 2015). 
No estudo (E9) de Azevedo e Kind (2013), o objetivo foi conhecer a atuação dos psicólogos das equipes dos NASF de Belo Horizonte (MG). As autoras apontaram que o NASF representa uma evolução do atendimento na $\mathrm{ABS}$, e que os psicólogos vêm redesenhando sua prática conforme a demanda da área da saúde. Os profissionais referiram não estar preparados e seguros para a atuação nesse campo, tendo relatado como desafios a elevada demanda de trabalho, as dificuldades para o trabalho com grupos, a integração com as equipes de saúde mental, assim como as limitações para a ampliação de sua atuação para além da clínica tradicional. Nesse sentido, apontaram a necessidade de, já no contexto de atuação, empreenderem uma revisão e ressignificação da própria trajetória formativa. Contudo, os psicólogos reconheceram que o NASF é um campo profícuo de estudos para melhorias na prática profissional e aperfeiçoamento da formação na área da saúde (Azevedo, \& Kind, 2013).

Em concordância, o estudo (E10) de Leite et al. (2013) também teve por objetivo analisar a inserção e a atuação da Psicologia na ABS, identificando possíveis desafios e potencialidades referidos por psicólogos dos NASF do município de Juazeiro do Norte (CE). As autoras referiram a existência de dificuldades no trabalho interdisciplinar e intersetorial, tendo-as atribuído ao predomínio do modelo biomédico e à inadequação dos serviços aos princípios do SUS. Todavia, consideraram que os psicólogos demonstraram motivação e disposição para criar possibilidades e estratégias de superação das dificuldades identificadas. Finalmente, as autoras apontaram o NASF como um espaço recente de atuação para a Psicologia, restando diversos aspectos a serem pesquisados diante da escassa produção científica sobre o tema (Leite et al., 2013).

Lancman et al. (2013), em seu estudo (E11), almejaram compreender as características da organização, das condições de trabalho e das vivências subjetivas relacionadas ao trabalho de dois NASF de São Paulo (SP). As autoras ponderaram que o trabalho dos NASF atendia aos princípios e diretrizes do SUS, delineando-se a partir da atuação de equipes com diversidade de recursos humanos, o que favorecia sua capacidade de resolutividade. Todavia, ressalvaram que características tradicionais da formação acadêmica, assim como a inexperiência profissional, representariam entraves para a atuação das equipes do NASF, implicando um "aprender fazendo", de acordo com as par- ticularidades de cada população e território. Nesse sentido, as autoras afirmaram que esse cenário, por um lado, favoreceria a flexibilização do trabalho às demandas da comunidade; por outro, em nível de sistema de saúde, limitaria a possibilidade de compartilhar e replicar experiências e ações bem-sucedidas, de forma a expandi-las para contextos mais amplos (Lancman et al., 2013).

No estudo E12, Archanjo e Schraiber (2012) declararam o objetivo de estudar a atuação de psicólogos em UBS de São Paulo (SP). As autoras realizaram uma análise institucional para compreender e problematizar a atuação dos psicólogos na saúde pública. Constataram que essa atuação segue as premissas do instituído e que, promovendo o novo, os psicólogos e as equipes de ESF poderiam refletir sobre essa instituição. Concluíram, pois, que os psicólogos não exerciam seu compromisso social de questionar o instituído e de criar espaços para os sujeitos serem instituintes, seguindo princípios da autonomia, corresponsabilidade, transversalidade de saberes e tendo como embasamento a Psicologia social comunitária, cuja ideologia ético-política e social pressupõe oportunizar que a comunidade tenha voz, consciência e se empodere de sua realidade (Archanjo, \& Schaiber, 2012).

Santos e Castro (2011) apresentaram um relato de experiência (E17), com o objetivo de retratar a vivência que tiveram em uma capacitação sobre processos de trabalho grupal das equipes das UBS de um município do interior de São Paulo. Concluíram que, por meio das dinâmicas utilizadas, foi possível descontruir a compreensão de que as técnicas de controle e obediência garantiriam a efetividade dos grupos. Os autores também referiram desenvolvimento da sensibilização, levantamento da problemática de grupo, estabelecimento de confiança e vínculo entre os participantes. Nessa perspectiva, segundo os autores, os profissionais estariam alinhados às diretrizes e aos princípios do SUS, cumprindo seu compromisso social (Santos, \& Castro, 2011).

Sundfeld (2010), por meio de um estudo de caso (E18), problematizou algumas demandas das equipes da ESF dirigidas à psicóloga de um NASF da cidade de São Paulo (SP), sua produção e implicações ético-políticas para o exercício da clínica, e os processos de subjetivação resultantes. A autora mapeou, nos discursos das equipes, as possíveis interlocuções e agenciamentos, traduzidos, para os profissionais da 
saúde, como demanda. Segundo a autora ponderou, as demandas, muitas vezes, expressam considerações morais, evidenciando concepções idealizadas de saúde e bem-estar. A título de conclusão, foi apresentada a proposição de que é preciso resistir aos mecanismos de disciplinarização da vida, o que pode ser conquistado a partir de uma postura dialógica, crítica e reflexiva das equipes, que contribua para a afirmação de formas de existir saudáveis e livres de padrões preestabelecidos (Sundfeld, 2010).

No estudo (E19) de Araujo et al. (2009), o objetivo foi relatar a experiência de um curso de extensão universitária sobre introdução à Psicologia destinado a um público não acadêmico. A experiência foi realizada por estudantes de pós-graduação da Universidade de São Paulo (USP), e o público foi formado por estudantes, donas-de-casa, educadoras aposentadas e um grupo de colegas de trabalho de uma cooperativa. As temáticas trabalhadas foram moradia, coletividade, objetos importantes (cama, bíblia, símbolos), sexualidade, loucura, sempre respeitando os conceitos e as experiências das pessoas sobre os temas, assim proporcionando um espaço para reflexão e criação de subjetividade. Segundo os autores, tomou-se "a Psicologia como uma ferramenta para escrever o mundo" (p. 29), uma estratégia inovadora para promover quebra de paradigmas sobre como trabalhar com grupos e pensar a subjetividade dos sujeitos (Araujo et al., 2009).

No estudo E20, Macedo e Dimenstein (2009) propuseram discutir concepções e práticas de cuidado de psicólogos no campo das políticas de saúde (SUS) e de assistência social (Sistema Único de Assistência Social - SUAS), no município de Natal (RN). Os autores abordaram peculiaridades da atuação dos profissionais da Psicologia, considerando haver uma tendência, no SUS, à intervenção especializada, fundamentada no saber instrumental, por meio do qual o profissional assume uma posição hierárquica condutora do cuidado. Já no SUAS, observaram uma atuação pautada por posturas de acolhimento e corresponsabilização, resultantes de reflexão sensível, em um exercício ético-político sobre a realidade. Em síntese, conforme afirmaram os autores, as práticas de cuidado produzidas pelos psicólogos no SUS e no SUAS transitam entre a adesão ao ideal de produzir sujeitos autônomos, produtivos e saudáveis - configurando-se como normatividade - e o movimento de buscar rupturas com a norma (Macedo, \& Dimenstein, 2009).
Ferreira (2008), em seu estudo (E22), se propôs a discutir sobre intervenção psicossocial e formação do psicólogo, a partir do estudo de caso de uma experiência de intervenção desenvolvida em uma escola do município Belo Horizonte (MG), como uma ação da ABS. O autor concluiu que a formação é a base para projetar, nos acadêmicos, a atuação nas diversas áreas e espaços da Psicologia. Nessa direção, o autor ressaltou a importância de uma formação que articule elementos da Psicologia clínica e da Psicologia social, promovendo o desenvolvimento de competências plurais para uma inserção profissional qualificada, independentemente da área atuação (Ferreira, 2008).

A partir dos autores e estudos que compuseram esta categoria temática - Atuação do psicólogo no SUS, no nível da Atenção Básica -, percebe-se que a Psicologia, no decorrer da história e com a evolução das políticas públicas, vem se reinventando quanto a sua atuação para atender às demandas desse campo. O mais recente cenário desvelado para a Psicologia, na ABS, é o NASF, que representa ainda um desafio tanto para os psicólogos quanto para os demais profissionais da área da saúde.

Especificamente no que diz respeito à atuação da Psicologia na ABS, a literatura tem evidenciado avanços e entraves. Os estudos analisados apontam que os psicólogos vêm atualizando suas práticas, ampliando sua inserção nas comunidades e diversificando suas estratégias de atuação. No entanto, ainda são relatadas situações-limite, em que prevalecem práticas tradicionais individualizantes, assim como práticas inovadoras ingênuas, que não revelam reflexão e compromisso ético-político e social. Convém destacar que, além destas questões, outras limitações emergiram da análise empreendida nesta categoria temática, como dificuldades na atuação em equipes multiprofissionais e interdisciplinares, especialmente quanto ao trabalho coletivo, que pressupõe o compartilhamento de saberes e favorece a clínica ampliada.

Cabe, ainda, salientar que, em muitos dos artigos que compuseram a síntese apresentada nesta categoria, observa-se uma abordagem que incorpora tanto a atuação quanto a formação em Psicologia. Essa constatação pode ser explicada pelo fato de que ambas têm interfaces importantes, visto que a atuação profissional é condicionada pela formação - não só acadêmica. Contudo, alguns autores, ao desenvolverem suas pesquisas, colocaram a ênfase na dimensão da 
atuação do profissional. Já outros focalizaram seus processos formativos. Assim, a seguir, é apresentada a síntese correspondente aos estudos cuja centralidade esteve no âmbito da formação do psicólogo.

\section{Formação do psicólogo para a atuação no SUS, no nível da Atenção Básica}

Frente aos desafios apontados na análise da atuação da Psicologia na ABS, fica reafirmada a importância de que os profissionais reconheçam novas possibilidades de se fazerem psicólogos nesse contexto. Segundo Cintra e Bernardo (2017), esses desafios e, por conseguinte, sua superação, esbarram nos processos de formação profissional. Nessa perspectiva, Oliveira et al. (2017) ponderam que a formação de psicólogos precisa rever a importância atribuída ao debate sobre política social e sobre a relação entre Psicologia e Saúde Pública, de forma a impactar, efetivamente, no campo das práticas (Oliveira et al., 2017). Nesta direção, revela-se pertinente conhecer a abordagem conferida à formação do psicólogo para a ABS na literatura sobre o tema. Com esse propósito, os artigos selecionados e que fundamentam essa categoria temática são: E5, E6, E8, E13, E14, E15, E16 e E21.

$\mathrm{O}$ estudo (E5) desenvolvido por Batista et al. (2014) teve o objetivo de caracterizar a formação em saúde no curso de Psicologia da Pontifícia Universidade Católica de Minas Gerais, considerando os cenários de aprendizagem e as dimensões de ensino, pesquisa e extensão, à luz das DCN. As autoras observaram que a proposta pedagógica do curso incorporava elementos da saúde, da saúde pública e da abordagem interdisciplinar, além de presença diversificada de cenários de aprendizagem. Constataram, no entanto, que os temas relativos à saúde estavam relativamente dispersos, que havia pouca articulação entre ensino-pesquisa-extensão e que a atuação multiprofissional e a abordagem interdisciplinar eram frágeis na realidade dos cenários de aprendizagem. Apesar desses entraves, as autoras concluíram que a proposta do curso avaliado ultrapassava as prescrições e assumia o currículo como possibilidade ampliada de formação, na perspectiva da construção de sujeitos. Ao finalizarem suas reflexões, ainda afirmaram a importância de fortalecer, na formação em Psicologia para a área da saúde, inserções integradas de ensino-pesquisa-extensão, disseminando modos de aprender e ensinar coeren- tes com as condições culturais, econômicas e políticas da sociedade (Batista et al., 2014).

Alves et al. (2014), no estudo E6, almejaram problematizar a formação profissional e suas lacunas para a atuação do psicólogo na saúde pública. Apresentaram uma proposta de estágio supervisionado, desenvolvida na Universidade Estadual da Paraíba, com fundamentação na Psicologia da Saúde, na Abordagem Centrada na Pessoa e na Política Nacional de Humanização. A proposta foi organizada por meio de intervenções nos três níveis de atenção - primário (ABS), secundário (Centro de Atenção Psicossocial) e terciário (alta complexidade) -, incluindo atividades de plantão de escuta psicológica, triagem, formação de grupos, diálogo multiprofissional com equipes do Centro de Referência de Assistência Social e do Centro de Referência Especializado de Assistência Social, grupos terapêuticos, orientação às famílias, atividades educativas, interconsultas com a equipe multiprofissional de saúde e mediação pedagógica junto a essa equipe. As autoras defenderam a relevância da atualização dos processos de formação, ampliando-os e tornando-os menos elitizados e mais engajados com as políticas públicas, especialmente no âmbito do SUS. Ao concluírem, argumentaram que, dessa forma, seria possível transformar as práticas psicológicas, despertando, nos estudantes, motivação para a atuação profissional nesse cenário, que tem aberto significativas oportunidades para a Psicologia (Alves et al., 2014).

O estudo (E8) de Hüning et al. (2013) discutiu a formação em Psicologia para a inserção no SUS, em Alagoas, por meio da análise dos currículos dos quatro cursos do estado. Os resultados indicaram predominância de abordagens psicodiagnósticas, com olhar centrado na doença e no atendimento individualizado. Contudo, a abordagem da integralidade foi observada em alguns currículos, evidenciando, nestes casos, potencial para a produção de transformações importantes na formação para o SUS. A esse respeito, as autoras concluíram que os cursos analisados atendiam às DCN, com diversificação dos cenários de ensino-aprendizagem, atuação multiprofissional e perfil generalista. Porém, destacaram que a ênfase colocada na clínica tradicional, diante das tímidas evidências de incorporação dos princípios do SUS, denunciava a valorização do modelo biomédico de formação (Hüning et al., 2013). 
Esses achados (E8) corroboram o estudo (E13) de Guareschi et al. (2012), que buscou analisar a formação em Psicologia, em seis cursos do estado do Rio Grande do Sul, em relação à demanda de formar profissionais para o SUS. Com base na análise das disciplinas da área biomédica, presentes nos currículos dos cursos, as autoras concluíram que havia uma fragmentação dos saberes, visto que conteúdos da psicopatologia, farmacologia, entre outros, não eram articulados ao campo da Psicologia. Ademais, estes conteúdos eram tratados sob perspectiva única, com privilégio da dimensão biológica do ser humano, resultando em uma abordagem dos conceitos de saúde e doença desconectada do princípio de integralidade do SUS. As autoras concluíram, ponderando que as concepções características do modelo biomédico, como neutralidade e objetividade, negam, em alguma medida, os princípios ampliados do SUS, que precisam ser assumidos nos processos de formação em Psicologia, no intuito de potencializar e mudar a realidade social (Guareschi et al., 2012).

No estudo E14, Santeiro (2012) declarou o objetivo de discutir o papel da Psicologia clínica no NASF, a partir da experiência de supervisão de estágios da Universidade Federal de Goiás. O autor observou que dificuldades relacionadas às condições de infraestrutura e de sobrecarga de trabalho impactaram supervisores e, especialmente, estagiários, repercutindo como impotência e frustração no contexto da construção de sua identidade profissional. Como conclusão, foi apontado que estudantes, supervisores locais (psicólogos) e supervisor acadêmico consideraram o estágio clínico no NASF um desafio para a formação de profissionais da saúde, processo sujeito a entraves ligados tanto às políticas de ensino superior quanto às de saúde pública (Santeiro, 2012).

Guareschi et al. (2011), em seu estudo (E15), tiveram o objetivo de analisar currículos de cursos de Psicologia do Rio Grande do Sul sob a perspectiva da formação de profissionais de saúde para o SUS. A partir da análise das disciplinas relacionadas aos conteúdos de Avaliação Psicológica, Psicopatologia e Psicoterapia, as autoras consideraram que a aproximação da Psicologia com a área da saúde implica uma perspectiva de estranhamento, visto que, enquanto a saúde se pauta nos princípios da integralidade, equidade e universalidade, os currículos dos cursos de Psicologia avaliados apresentaram-se transversalizados pelo paradigma da psicopatologia, focados no diagnóstico da doença, organizados de modo linear e assumindo a avaliação psicológica como normatividade, na lógica de um saber técnico privativo do psicólogo. Tais fatores, na avaliação das autoras, ao priorizarem o "psiquismo", distanciam os profissionais da demanda e da visão do SUS, que se apoia no trabalho interdisciplinar (Guareschi et al., 2011).

O estudo (E16) de Gonze e Silva (2011) expressou o interesse de analisar a percepção dos docentes de graduação da Universidade Federal de Juiz de Fora (MG) sobre a formação para a integralidade como princípio do SUS, contando com a participação do coordenador e de quatro professores do curso de Psicologia. As autoras, inicialmente, problematizaram o conceito de integralidade, apontando-o como polissêmico. Prosseguindo, referiram que, no contexto da pesquisa desenvolvida, constataram que a integralidade foi percebida pelos professores como o principal valor do bom profissional de saúde. Ainda observaram que os professores expressaram um entendimento da integralidade como processo em construção, com implicação de estudantes, usuários e professores. À guisa de conclusão, as autoras afirmaram que o movimento de conquista da integralidade favorece as mudanças necessárias na formação em saúde, contribuindo para a construção de um conhecimento transformador da realidade (Gonze, \& Silva, 2011).

O estudo E21, de Prates e Nunes (2009), apresentou uma reflexão sobre o lugar do psicólogo na saúde pública como um campo do saber em (re)construção. Para tanto, os autores problematizaram a concepção de saúde vigente e a de Psicologia na saúde pública, discutindo suas possibilidades, por meio de uma experiência de estágio, realizada enquanto estudantes da Pontifícia Universidade Católica de Minas Gerais. Os autores relataram ser comum que os psicólogos, na ABS, pautem suas práticas pelos pressupostos da clínica tradicional. Contudo, ponderaram que a prática de estágio vivenciada avançou para além desse modelo, incorporando intervenções diretamente com os usuários, inseridos em sua realidade, e contribuindo, assim, para o seu bem-estar. Concluindo, os autores fizeram a ressalva de que o restrito contingente de psicólogos na saúde pública pode ser um limitador para a produção de práticas inovadoras nesse campo (Prates, \& Nunes, 2009).

Quanto à Formação do psicólogo para a atuação no SUS, no nível da Atenção Básica, a análise dos estudos selecionados evidenciou que há gran- 
des dificuldades a serem enfrentadas para que se conquiste uma formação alinhada aos princípios do SUS. Os estudos apontaram na direção da manutenção, ainda, de processos formativos que reforçam a atuação segundo o modelo biomédico hegemônico. Nesse sentido, a formação em Psicologia revela-se perpassada pelo paradigma da clínica tradicional e por uma concepção de saúde como normalidade, perspectiva que orienta a inserção dos estudantes nos cenários de práticas de ensino-aprendizagem. Todavia, há estudos que demonstram que a Psicologia está buscando sair da sua zona de conforto, utilizando práticas, teorias e abordagens menos lineares e pragmáticas.

\section{Considerações Finais}

O interesse em compreender como a literatura científica do período de 2006 a 2016 aborda a Psicologia no SUS, no nível da Atenção Básica, guiou esta revisão. No percurso, nos deparamos com a trajetória histórico-social, política e econômica da inserção da Psicologia nesse contexto, no qual ainda hoje continua a adentrar. Com base na análise apresentada, evidencia-se que o foco e a prioridade das pesquisas estão colocados na dimensão da atuação do psicólogo. Acredita-se que isso possa ser explicado pelo fato de que o NASF é uma proposta relativamente recente. Além disso, foi observada, em diversos estudos, certa dificuldade frente a esse novo modelo de atuação que requer, da Psicologia, um desprender-se das abordagens características da área clínica -, restando clara a necessidade de um olhar mais histórico-social, que desvele a subjetividade de cada sujeito.

Apesar da ênfase dada à atuação do psicólogo, pondera-se que ela constitui um reflexo intrínseco à formação acadêmica, que ainda faz perdurar modelos e eixos biomédicos psicopatológicos no ensino-aprendizagem, em detrimento de currículos (instituídos e instituintes) que efetivamente incentivem e favoreçam o compromisso dos profissionais com o SUS e a compreensão da construção social da subjetividade e da identidade do sujeito e do profissional. Argumenta-se que a formação teria condições de incentivar outras possibilidades, fortalecendo a Psicologia enquanto campo de saberes e práticas implicados em um compromisso ético, político e social. Nesse sentido, uma formação que valorize a pluralidade teórico-metodológica surge como alternativa importante, incluindo, por exemplo, a ótica da análise institucional e da Psicologia social comunitária, entre outras, ao mesmo tempo relativizando a ênfase nas abordagens clínicas tradicionais. Contudo, pergunta-se: a formação está a serviço de quê? Reforçando quais ideologias?

Frente a esses questionamentos, cabe a proposição de que os sujeitos envolvidos com os processos de formação assumam, também, o compromisso com uma perspectiva crítico-reflexiva e problematizadora. Assim, mesmo que a realidade do campo da saúde pública, enquanto cenário de ensino-aprendizagem e de práticas profissionais, não ofereça as condições idealizadas pela Reforma Sanitária e pelo SUS, o desenvolvimento de competências de crítica e reflexão representa um recurso para a criação de novos fazeres, que se constituam como vias de resistência e superação dos desafios.

Há que se considerar que a ABS, enquanto espaço de inserção de psicólogos no SUS, sofre os efeitos de pressões de diferentes naturezas: a demanda pelo profissional é grande e crescente, o número de profissionais frente a essa demanda é insuficiente, os gestores da saúde avaliam a atuação do profissional como medida de produtividade (entendida como número de atendimentos). Todos esses elementos induzem à manutenção de uma ordem em que prevalece o atendimento clínico individualizado, que responde, também, em muitas situações, às expectativas dos usuários. Em se perpetuando essa dinâmica, corre-se o risco de que a Psicologia, na ABS - seja na ESF ou no NASF - se consolide, apenas, como prática especializada. Para romper com esse estado de coisas, é importante atuar em ambas as frentes: a da atuação e a da formação.

No âmbito da atuação, algumas experiências têm demonstrado a viabilidade e a importância de que o psicólogo, ao refletir sobre seu trabalho nesse lugar que é a ABS, reinvente seu fazer, em um movimento de articulação com as equipes em que está inserido, construindo um trabalho interdisciplinar, que seja de suporte às equipes, mas que também seja de promoção da saúde junto aos usuários. Um trabalho que ultrapasse os limites dos consultórios, de forma que 0 profissional se reconheça, também, atuando com as comunidades, nos lugares em que as pessoas vivem suas vidas cotidianamente. Já no âmbito da formação, com vistas ao desenvolvimento de um egresso para construir essas novas práticas, surge essencial o fortalecimento de uma educação crítica, fundamentada 
em metodologias problematizadoras, promotora de competências colaborativas para o trabalho interdisciplinar e com inserção qualificada em cenários reais de ensino-aprendizagem.

Ressalta-se, assim, a importância de que a Psicologia, para além de repensar sua formação e sua atu- ação, se engaje, também, na produção e socialização de novos conhecimentos sobre essas temáticas, haja vista que tanto o compartilhamento e a replicação de experiências exitosas quanto o aprendizado promovido pelos insucessos eventuais podem ser potentes propulsores de mudanças.

\section{Referências}

Alves, R. F., Gaião, E. S., Santos, G. C., \& Soares, L. M. R. (2014). Proposta de estágio supervisionado para atuação de psicólogos na saúde pública. Revista da Abordagem Gestáltica, 20(1), 21-30.

Archanjo, A. M., \& Schraiber, L. B. (2012). A atuação dos psicólogos em unidades básicas de saúde na cidade de São Paulo. Saúde e Sociedade, 21(2), 351-363. https:// doi.org/10.1590/S0104-12902012000200009

Araujo, T. W., Almeida, M. M., \& Guimaraes, D. S. (2009). Reflexões sobre o ensino de psicologia fora da academia a partir de um curso destinado à comunidade. TransFormações em Psicologia, 2(2), 8-31.

Azevedo, N. S., \& Kind, L. (2013). Psicologia nos núcleos de apoio à saúde da família em Belo Horizonte. Psicologia: Ciência e Profissão, 33(3), 520-535. https://doi.org/10.1590/S1414-98932013000300002

Batista, C. B., Carmona, D. S., \& Fonseca, S. L. (2014). Formação em saúde e os cenários de aprendizagem no curso de psicologia. Psicologia Argumento, 32(78), 17-25. https://doi.org/10.7213/psicol.argum.32.078.DS02

Carvalho, Y. M., \& Ceccim, R. B. (2009). Formação e educação em saúde: Aprendizados com a saúde coletiva. In G. W. S. Campos, M. C. S. Minayo, M. Akerman, M. Drumond Júnior, \&Y. M. Carvalho (Orgs.), Tratado de saúde coletiva (2a ed., Cap. 5, pp. 137-170). São Paulo, SP: Hucitec.

Cela, M., \& Oliveira, I. F. (2015). O psicólogo no núcleo de apoio à saúde da família articulação de saberes e ações. Estudos de Psicologia (Natal), 20(1), 31-39. https://doi.org/10.5935/1678-4669.20150005

Cintra, M. S., \& Bernardo, M. H. (2017). Atuação do psicólogo na atenção básica do SUS e a psicologia social. Psicologia: Ciência e Profissão, 37(4), 883-896. https://doi.org/10.1590/1982-3703000832017

Costa, N. R., Siqueira, S. V., Uhr, D., Silva, P. F., \& Molinaro, A. A. (2011). Reforma psiquiátrica, federalismo e descentralização da saúde pública no Brasil. Ciência \& Saúde Coletiva, 16(12), 4603-46014. https://doi.org/10.1590/ S1413-81232011001300009

Ferreira, J. L., Neto (2008). Intervenção psicossocial em saúde e formação do psicólogo. Psicologia \& Sociedade, 20(1), 62-69. https://doi.org/10.1590/S0102-71822008000100007

Ferreira, J. L., Neto (2011). Psicologia, políticas públicas e o SUS. Belo Horizonte, MG: Autêntica.

Guareschi, N. M. F., Reis, C., Dhein, G., Bennemann, T., \& Machry F. C. (2011). A avaliação psicológica, psicopatologia e as psicoterapias na formação do profissional de saúde para o SUS: Um estudo dos currículos dos cursos de psicologia. Revista Mal-Estar e Subjetividade, 21(1), 171-204.

Guareschi, N. M., Machry F. C., Reis, C., Dhein, G., \& Bennemann, T. (2012). Implicações das áreas biomédicas da formação do psicólogo no SUS. Psicologia Argumento, 30(70), 503-513. https://doi.org/10.7213/psicol. argum.6137

Gomes, C. A. V., \& Pedrero, J. N. (2015). Queixa escolar: Encaminhamentos e atuação profissional em um município do interior paulista. Psicologia: Ciência e Profissão, 35(4), 1239-1256. https://doi.org/10.1590/19823703002192013

Gonze, G. G., \& Silva, G. A. (2011). A integralidade na formação dos profissionais de saúde: Tecendo valores. Physis: Revista de Saúde Coletiva, 21(1), 129-146. https://doi.org/10.1590/S0103-73312011000100008

Hüning, S. M., Silva, A. K., Silva, W. V. N., \& Lessa, F. M. S. (2013). Formação para SUS nos cursos de psicologia em alagoas. Psicologia em Estudo, 18(1), 157-167. https://doi.org/10.1590/S1413-73722013000100016

Jimenez, L. (2011). Psicologia na atenção básica à saúde: Demanda, território e integralidade. Psicologia \& Sociedade, 23(esp): 129-139. https://doi.org/10.1590/S0102-71822011000400016 
Lancman, S., Gonçalves, R. M. A., Cordone, N. G., \& Barros, J. O. (2013). Estudo do trabalho e do trabalhar no núcleo de apoio à saúde da família. Revista de Saúde Pública, 47(5), 968-975. https://doi.org/10.1590/S00348910.2013047004770

Leite, D. C., Andrade, A. B., \& Bosi, M. L. M. (2013). A inserção da psicologia nos núcleos de apoio à saúde da família. Physis: Revista de Saúde Coletiva, 23(4), 1167-1187. https://doi.org/10.1590/S0103-73312013000400008

Leite, D. F., Nascimento, D. D. G., \& Oliveira, M. A. C. (2014). Qualidade de vida no trabalho de profissionais do NASF no município de São Paulo. Physis: Revista de Saúde Coletiva, 24(2), 507-525. https://doi.org/10.1590/ S0103-73312014000200010

Macedo, J. P., \& Dimenstein, M. (2009). Psicologia e a produção do cuidado no campo do bem-estar social. Psicologia \& Sociedade, 21(3), 293-300. https://doi.org/10.1590/S0102-71822009000300002

Manoel, R. A., Combinato, D. S., Gomes, F. M. A., \& Silva, K. F. (2014). O papel do trabalho e da formação acadêmica no projeto profissional do trabalhador da saúde. Trabalho, Educação e Saúde, 12(3), 595-614. https://doi. org/10.1590/1981-7746-sip00008

Ministério da Saúde. (2009). Programa nacional de reorientação da formação profissional em saúde pró-saúde: Objetivos, implementação e desenvolvimento potencial. Brasília, DF: o autor. Recuperado de http://bvsms.saude. gov.br/bvs/publicacoes/programa_nacional_reorientacao_profissional_saude.pdf

Oliveira, I. F., \& Amorim, K.M.O. (2011). O SUS como desafio para a formação em psicologia. Psicologia: Ciência e Profissão, 31(2), 340-357. https://doi.org/10.1590/S1414-98932011000200011

Oliveira, I. F., Amorim, K. M. O., Paiva, R. A., Oliveira, K. S. A., Nascimento, M. N. C., \& Araújo, R. L. (2017). A atuação do psicólogo nos NASF: Desafios e perspectivas na atenção básica. Temas em Psicologia, 25(1), 291-304. https:// doi.org/10.9788/TP2017.1-17Pt

Prates, L. G., \& Nunes, L. P. (2009). A (re)construção do lugar do psicólogo na saúde pública: Das quatro paredes do centro de saúde para os lares. Pesquisas e Práticas Psicossociais, 4(1), 96-101.

Perrella, A. C. (2015). A experiência da psicologia no NASF: Capturas, embates e invenções. Gerais: Revista Interinstitucional de Psicologia, 8(2), 443-452.

Portaria SAS/MS n. 224, de 29 de janeiro de 1992. Estabelece diretrizes e normas. Recuperado de: http:/ /www.saude. mg.gov.br/images/documentos/Portaria_224

Portaria GM n. 154, de 24 de janeiro de 2008. Cria os Núcleos de Apoio à Saúde da Família - NASF. Recuperado de http://189.28.128.100/dab/docs/legislacao/portaria154_24_01_08.pdf

Portaria MS n.2.436, de21 de setembro de 2017. Aprova a política nacional de atenção básica, estabelecendo a revisão de diretrizes para a organização da atenção básica, no âmbito do Sistema Único de Saúde (SUS). Recuperado de http://bvsms.saude.gov.br/bvs/saudelegis/gm/2017/prt2436_22_09_2017.html

Santeiro, T. V. (2012). Processos clínicos em núcleos de apoio à saúde da família/NASF: Estágio supervisionado. Psicologia: Ciência e Profissão, 32(4), 942-955. https://doi.org/10.1590/S1414-98932012000400013

Sousa, D., Oliveira, I. F., \& Costa, A. L. F. (2015). Entre o especialismo e o apoio: Psicólogos no núcleo de apoio à saúde da família. Psicologia USP, 26(3), 474-483. https://doi.org/10.1590/0103-656420140059

Souza, L. V., \& Santos, M. A. (2012). Processo grupal e atuação do psicólogo na atenção primária à saúde. Journal of Human Growth and Development, 22(3), 388-395. https://doi.org/10.7322/jhgd.46711

Santos, A. O., \& Castro, E. O. (2011). Demanda por grupos, psicologia e controle. Psicologia e Sociedade, 23(2), 325331. https://doi.org/10.1590/S0102-71822011000200013

Scarcelli, I. R., \& Junqueira, V. (2011). O SUS como desafio para a formação em psicologia. Psicologia: Ciência e Profissão, 31(2), 340-357. https://doi.org/10.1590/S1414-98932011000200011

Soratto, J., Pires, D. E. P., Dornelles, S., \& Lorenzetti, J. (2015). Estratégia saúde da família: Uma inovação tecnológica em saúde. Reflexão, 24(2), 584-592. https:// doi.org/10.1590/0104-07072015001572014

Sundfeld, A. C. (2010). Clínica ampliada na atenção básica e processos de subjetivação: Relato de uma experiência. Physis: Revista de Saúde Coletiva, 20(4), 1079-1097. https://doi.org/10.1590/S0103-73312010000400002

http://www.scielo.br/pdf/physis/v20n4/a02v20n4.pdf 


\section{Raquel Antunes de Mello}

Mestra em Ciências da Saúde da Universidade Comunitária da Região de Chapecó (Unochapecó), Chapecó SC. Brasil.

E-mail: quelmello@unochapeco.edu.br

(D) http://orcid.org/0000-0001-6428-7635

Carla Rosane Paz Arruda Teo

Docente do Programa de Pós-Graduação em Ciências da Saúde da Universidade Comunitária da Região de Chapecó (Unochapecó), Chapecó - SC. Brasil.

E-mail: carlateo@unochapeco.edu.br

(iD) http://orcid.org/0000-0002-1534-6261

Endereço para envio de correspondência:

Rua Marechal José Bormann, 451 D. Apto 301.

CEP: 89801-050. Centro. Chapecó - SC. Brasil.

Recebido 13/10/2017

Reformulado 26/06/2018 - 23/07/2018

Aceito 10/09/2018

Received 10/13/2017

Reformulated 06/26/2018 - 07/23/2018

Approved 09/10/2018

Recibido 13/10/2017

Reformulado 26/06/2018 - 23/07/2018

Aceptado 10/09/2018

Como citar: Mello, R. A.; Teo, C. R. P. A. (2019). Psicologia: entre a atuação e a formação para o Sistema Único de Saúde. Psicologia: Ciência e Profissão, 39, 1-16. https://doi.org/10.1590/1982-3703003186511

How to cite: Mello, R. A.; Teo, C. R. P. A. (2019).Psychology: between practice and training for the Unified Health System. Psicologia: Ciência e Profissão, 39, 1-16. https://doi.org/10.1590/1982-3703003186511

Cómo citar: Mello, R. A.; Teo, C. R. P. A. (2019). Psicología: entre la práctica y la capacitación para el Sistema Único de Salud Psicologia: Ciência e Profissão, 39, 1-16. https://10.1590/1982-3703003186511 